\title{
Communications
}

\section{Selective Detection of Serotonin from the Interference by Ascorbic Acid and Uric Acid at Poly(thionine)-modified Glassy Carbon Electrode}

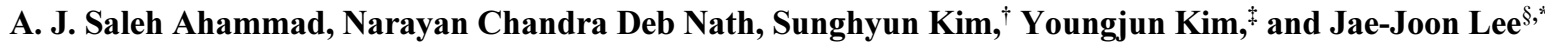 \\ Department of Advanced Technology Fusion, Konkuk University, Seoul 143-701, Korea \\ ${ }^{\dagger}$ Department of Bioscience and Biotechnology, Konkuk University, Seoul 143-701, Korea \\ *Department of Applied Biochemistry, Konkuk University, Chungju 380-701, Korea \\ ${ }^{\S}$ Department of Applied Chemistry, Konkuk University, Chungju 380-701, Korea. *E-mail: jjlee@kku.ac.kr \\ Received November 19, 2010, Accepted January 19, 2011
}

Key Words : Selective detection, Poly(thionine)-modified electrode, Serotonin, Cyclic voltammetry

Serotonin (5-hydroxytryptamine, 5-HT) is one of the important neurotransmitters in brain. ${ }^{1}$ Much attentions have been paid on the detection of 5-HT and electrochemical methods are preferable among the traditional methods, due to their simplicity and high sensitivity, for the detection of 5$\mathrm{HT}^{2,3}$ However, the interference by co-existing ascorbic acid (AA) and uric acid (UA) is one of the major problems in the electrochemical sensing of 5-HT.

Various approaches, such as using polymer film modified electrode, ${ }^{4}$ applying chemical modification, ${ }^{5}$ employing nano materials and molecularly imprinted polymers, ${ }^{3,6}$ have been developed to detect 5-HT selectively from interferences. The polymer-modified electrodes have widely been used because of their enhanced selectivity and sensitivity for many analytes. ${ }^{7}$ Previously, we have reported the role of poly(thionine) film $(\mathrm{PTH})$ deposited on the electrochemically preanodized glassy carbon electrode (GCE) for the separation of the voltammetric signal of dopamine (DA) from that of AA and UA. ${ }^{8}$ In this communication, we are presenting the preliminary results of the electrochemical signal separation of 5-HT by suppressing those of AA and UA on another type PTH modified GCE (PTHGCE) which is prepared by electrochemical deposition of PTH directly on the mechanically polished GCE.

The electrochemical polymerization process of thionine by cyclic voltammetry (CV) in a phosphate buffer solution (PBS, pH 7.0) containing $0.5 \mathrm{mM}$ thionine is shown in Figure 1. It is known that cationic monomers of thionine, essential for electrochemical polymerization of thionine, are formed at the potentials higher than $0.8 \mathrm{~V} v s$. $\mathrm{Ag} / \mathrm{AgCl}{ }^{9}$ When the potential was lower than this value, only the redox peak for the monomer was seen and no sign of further polymerization was observed (inset of Figure 1). Here we applied wide potential range for sweeping (from -0.4 to 1.1 $\mathrm{V})$ to induce the electrochemical polymerization on the surface of GCE. At the very first potential sweep, only one redox peak for the monomer was seen at $c a$. $-0.18 \mathrm{~V}$, where- as the second peak was developed at $c a .0 .1 \mathrm{~V}$ after the second sweep. It kept growing on successive sweeping while the first peak was not growing anymore as reported in literature. 9 The monomeric cation was expected to be formed during the first potential sweep at or above $0.8 \mathrm{~V}$ and it caused the following electrochemical polymerization, which was responsible for the growth of the second redox peak. This result indicated the growth of PTH, with light violet color, on the GCE surface to form PTHGC.

Figure 2 shows the CVs of AA, UA, and 5-HT, respectively, along with that obtained from the mixture $(50,50$, and $10 \mu \mathrm{M}$, respectively) in $0.1 \mathrm{M}$ PBS (pH 7.0) at bare GCE. It is obvious that AA, UA, and 5-HT are electrochemically active at bare GCE. ${ }^{4,8}$ The anodic oxidation peaks were observed at ca. $0.32,0.43$, and $0.43 \mathrm{~V}$ for AA, UA, and 5HT, respectively (Figure 2, ii, iii, iv). However, only a single oxidation peak was observed at $c a .0 .48 \mathrm{~V}$ from the mixture

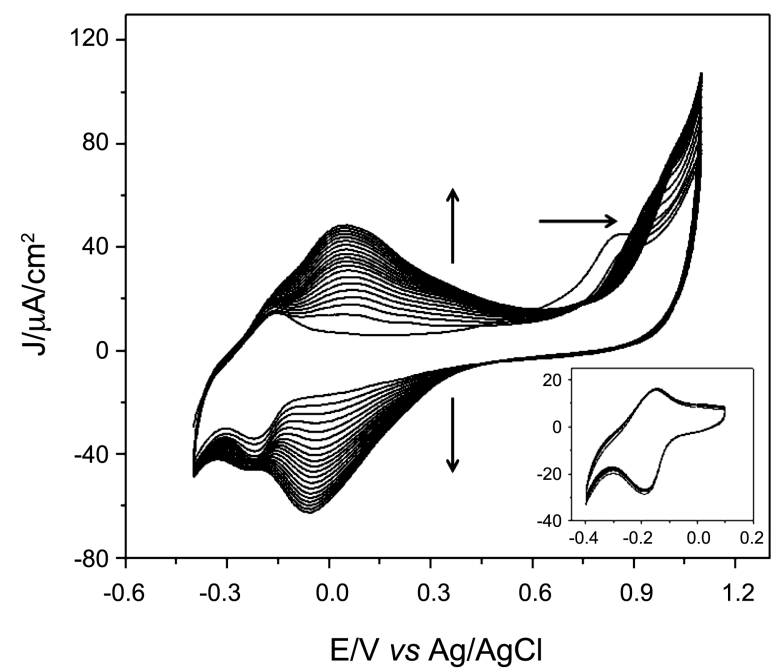

Figure 1. CVs for the electropolymerization of $0.5 \mathrm{mM}$ thionine in $0.1 \mathrm{M} \mathrm{pH}$ 7.0 PBS at GCE. Inset shows CV of thionine without polymerization. 


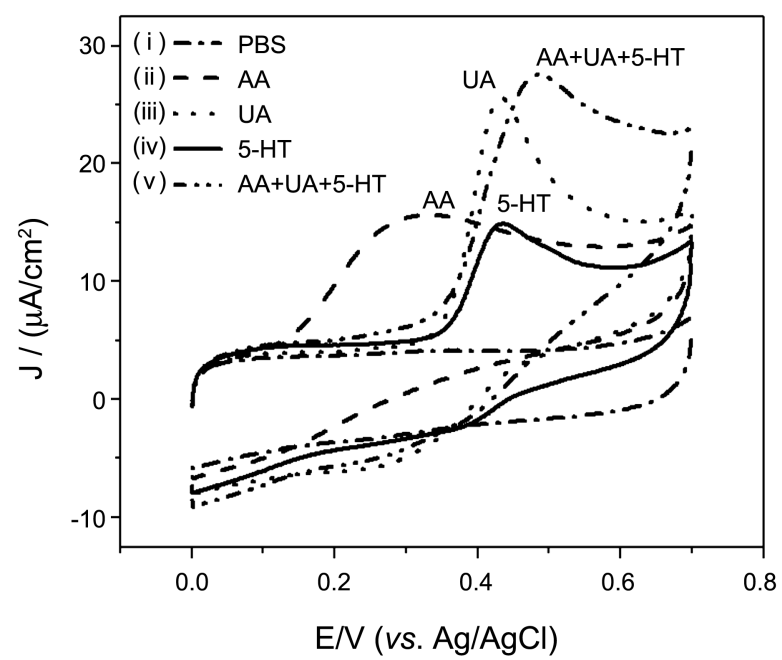

Figure 2. CVs of blank PBS (i); $50 \mu \mathrm{M}$ AA (ii); $50 \mu \mathrm{M}$ UA (iii); $10 \mu \mathrm{M} 5-\mathrm{HT}$ (iv) and a mixture of $50 \mu \mathrm{M} \mathrm{AA}, 50 \mu \mathrm{M}$ UA, and 10 $\mu \mathrm{M} 5-\mathrm{HT}(\mathrm{v})$ at bare GCE. Scan rate: $50 \mathrm{mV} / \mathrm{s}$.

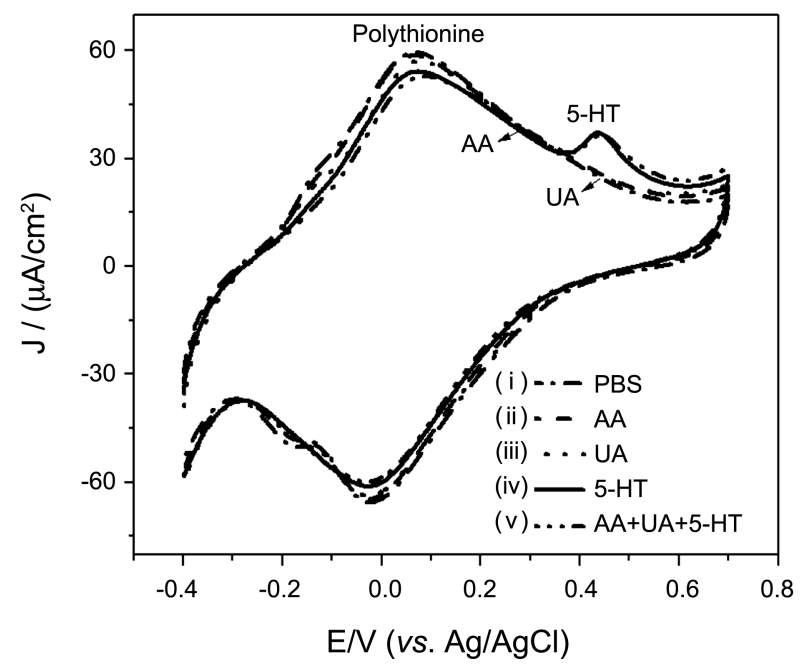

Figure 3. CVs of blank PBS (i); $50 \mu \mathrm{M}$ AA (ii); $50 \mu \mathrm{M}$ UA (iii); $10 \mu \mathrm{M} 5-\mathrm{HT}$ (iv) and a mixture of $50 \mu \mathrm{M}$ AA, $50 \mu \mathrm{M}$ UA, and 10 $\mu \mathrm{M}$ 5-HT (v) at PTHGC. Scan rate: $50 \mathrm{mV} / \mathrm{s}$.

solution (Figure 2, v). This indicates that 5-HT cannot be resolved from the AA and UA at the bare GCE. On the other hand, the signals from AA and UA were almost completely suppressed at the PTHGC as shown in Figure 3 (ii, iii), even if the concentrations of them $(50 \mu \mathrm{M})$ were 5 times higher than that of 5-HT $(10 \mu \mathrm{M})$. A very small response corresponds to the oxidation of UA was detected when the concentration of UA was higher than $200 \mu \mathrm{M}$ but it was still insignificant. AA did not show any traceable response even at the concentration range up to $5 \mathrm{mM}$ (data not shown). The rationale of the signal suppression is unclear at this stage and it is still under investigation. It is very likely that the electron-rich nitrogen atom of the PTH film hinders the anionic AA and UA in a PBS at $\mathrm{pH} 7.0$ from approaching to the PTHGC surface. However, one anodic oxidation peak was clearly observed at ca. $0.43 \mathrm{~V}$ for 5 -HT (Figure 3, iv). Furthermore, this peak overlapped with the oxidation wave obtained from

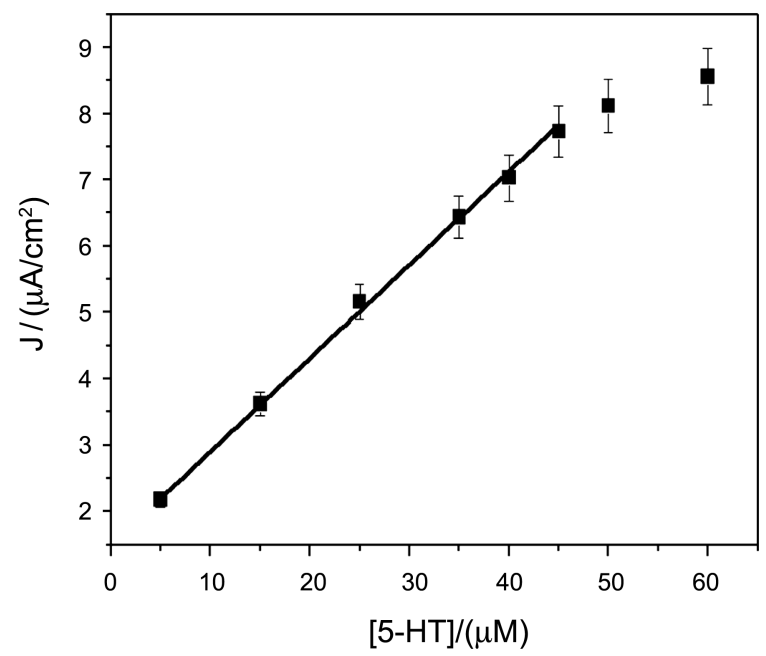

Figure 4. Calibration plots for 5-HT in $0.1 \mathrm{M} \mathrm{pH} 7.0$ PBS containing $50 \mu \mathrm{M}$ of both AA and UA.

the solution containing AA, UA, and 5-HT together (Figure $3, \mathrm{v})$. This clearly indicated that the peak observed from the mixture could be attributed solely to the oxidation of 5-HT with no interference by AA and UA at the PTHGC. The response of 5-HT might be attributed to the strong hydrogenbonding between the analyte and nitrogen atoms on PTHGC.

To verify the applicability of the PTHGC for the selective determination of 5-HT, we investigated the current responses of 5-HT at various concentrations in the presence of $50 \mu \mathrm{M}$ of both AA and UA, respectively. 5-HT showed linear responses, independently, in the concentration range up to ca. $45 \mu \mathrm{M}$, with the correlation coefficient $\left(\mathrm{R}^{2}\right)$ of 0.99 along with the detection limit (3s) of $0.3 \mu \mathrm{M}$, as shown in Figure 4. The linear range and detection limit of 5-HT are comparable to the values reported elsewhere. ${ }^{1,6}$

In this communication, we have shown that the newly processed PTHGC can separate the electrochemical response of 5-HT from the interferences of AA and UA. Further useful applications of this electrode for the simultaneous detection of DA and 5-HT in the presence of AA and UA are under investigation in our laboratory.

Acknowledgments. This work was supported by Konkuk University in 2010.

\section{References}

1. Oni, J.; Nyokong, T. Anal. Chim. Acta 2001, 434, 9.

2. Ahammad, A. J. S.; Sarker, S.; Rahman, M. A.; Lee, J. J. Electroanalysis 2010, 22, 694.

3. Ahammad, A. J. S.; Lee, J. J.; Rahman, M. A. Sensors 2009, 9, 2289.

4. Selvaraju, T.; Ramaraj, R. Electrochem. Commun. 2003, 5, 667.

5. Zen, J. M.; Chen, I. L.; Shih, Y. Anal. Chim. Acta 1998, 369, 103.

6. Santos, W. D. J. R.; Lima, P. R.; Tarley, C. R. T.; Höehr, N. F.; Kubota, L. T. Anal. Chim. Acta 2009, 631, 170.

7. Kumar, S. A.; Tang, C. F.; Chen, S. M. Talanta 2008, 74, 860.

8. Yuan, Y.; Ahammad, A. J. S.; Xu, G. R.; Kim, S.; Lee, J. J. Bull. Korean Chem. Soc. 2008, 29, 1883.

9. Gao, Q.; Cui, X.; Yang, F.; Ma, Y.; Yang, X. Biosens. Bioelectron. 2003, 19, 277. 